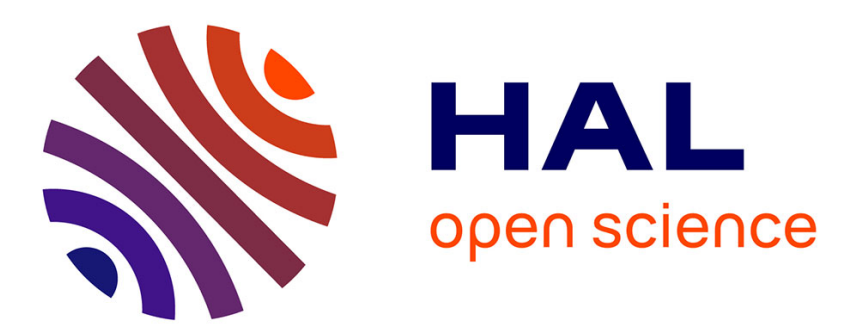

\title{
Target Transmission Radius over LMST for Energy-Efficient Broadcast Protocol in Ad Hoc Networks.
}

François Ingelrest, David Simplot-Ryl, Ivan Stojmenovic

\section{- To cite this version:}

François Ingelrest, David Simplot-Ryl, Ivan Stojmenovic. Target Transmission Radius over LMST for Energy-Efficient Broadcast Protocol in Ad Hoc Networks.. The 39th IEEE International Conference on Communications (ICC'04)., Jun 2004, Paris, France. inria-00000033

\section{HAL Id: inria-00000033 \\ https://hal.inria.fr/inria-00000033}

Submitted on 18 May 2005

HAL is a multi-disciplinary open access archive for the deposit and dissemination of scientific research documents, whether they are published or not. The documents may come from teaching and research institutions in France or abroad, or from public or private research centers.
L'archive ouverte pluridisciplinaire HAL, est destinée au dépôt et à la diffusion de documents scientifiques de niveau recherche, publiés ou non, émanant des établissements d'enseignement et de recherche français ou étrangers, des laboratoires publics ou privés. 


\section{Target Transmission Radius over LMST for Energy-Efficient Broadcast Protocol in Ad Hoc Networks}

\author{
François Ingelrest and David Simplot-Ryl \\ IRCICA/LIFL, INRIA futurs \\ University of Lille 1, France \\ Email: \{Francois.Ingelrest, David.Simplot\}@lifl.fr
}

\author{
Ivan Stojmenović \\ Computer Science, SITE, \\ University of Ottawa, Canada \\ Email: ivan@site.uottawa.ca
}

\begin{abstract}
We investigate minimum energy broadcasting problem where mobile nodes have the capability to adjust their transmission range. The power consumption for two nodes at distance $r$ is $r^{\alpha}+c$, where $\alpha \geq 2$ and $c$ is a constant that includes signal processing and minimal reception power. We show that, for $c>0$ (which is realistic assumption), it is not optimal to minimize transmission range. Furthermore, we demonstrate that there exists an optimal radius, computed with an hexagonal tiling of the network area, that minimizes the power consumption. For $\alpha>2$ and $c>0$, the optimal radius is $r=\sqrt[\alpha]{\frac{2 c}{\alpha-2}}$, which is derived theoretically, and confirmed experimentally. We propose also a localized broadcast algorithm $T R-L B O P$ which takes this optimal radius into account. This protocol is experimentally shown to be efficient compared to existing localized protocol $L B O P$ and globalized $B I P$ protocol. Most importantly, TR-LBOP is shown to have limited energy overhead with respect to $B I P$ for all network densities, which is not the case with $L B O P$ whose overhead explodes for higher densities.
\end{abstract}

\section{INTRODUCTION}

Wireless ad hoc networks are formed by a set of hosts that operate in a self-organized and decentralized manner, forming a dynamic autonomous network where communications take place over a wireless channel. Due to propagation path loss, transmission radii are limited, leading to a multi-hop routing where intermediate hosts between two communicating nodes act as routers.

In a broadcasting task, also referred to as flooding, a source node sends the same information to all the other hosts in the network. The easiest solution to achieve this is known as blind flooding, in which every node retransmits the message when it is received for the first time. This elementary protocol leads to a lot of duplicated packets and is inefficient from an energetic point of view. In this paper, we consider the minimum energy broadcasting problem in which the global energy consumption has to be minimized, while still guaranteeing the full coverage of the network.

Several solutions have already been proposed to reduce the needed number of transmitting nodes, but we especially focus on energy-efficient broadcast protocols, where nodes can choose and modify their transmission power. Indeed, although radii cannot exceed a given limit, they can be shorter than this maximum value, as energy consumption highly depends on the chosen radius. Most of the existing solutions are globalized, that is, each node requires global information about the network to choose its radius for a given transmission. This leads to an unacceptable communication overhead, due to information update, and unusable protocols in a practical context. Obviously, it is preferable in ad hoc networks with limited devices to consider localized protocols, in which nodes make decisions based solely on the knowledge of their 1-hop or 2-hops neighborhood.

In the literature, there exists several protocols to optimize the energy consumption by adjusting transmission powers. We distinguish here two families: topology control oriented protocols and broadcast oriented protocols:

- The first one computes the transmission power for each node such that the network is still connected, independently of how the broadcast will occur. Hence, every node can be the source of a broadcast and is able to reach any other node. Most of these protocols are based on the Minimum Spanning Tree (MST) and are globalized, since the computing of the MST needs global information about the network. Recently, localized protocols have been proposed [1]-[3], mainly based on the Relative Neighborhood Graph (RNG) [4] and the Local Minimum Spanning Tree (LMST) [5].

- The second family achieves the same goal, but considers a particular instance of a broadcast, i.e. from a given source node. The most efficient heuristic, called Broadcast Incremental Power (BIP) [6], constructs a tree starting from the source node and adds new nodes one at time according to a cost evaluation. This protocol is a globalized one, and cannot be applied in real ad hoc networks with independent nodes.

In this paper we are interested in broadcast oriented protocols using omnidirectional antennas in wireless ad hoc networks. We propose a localized protocol based on the idea that there exists an optimal radius, computed by using an hexagonal tiling of the network area. We first show that this optimal range exists and can be computed theoretically. Then we present 
our protocol, named Target Radius LMST Broadcast Oriented Protocol (TRLBOP), which is based on a modified version of $\angle B O P$ [2] to take into account the existence of an optimal radius. We show that the resulting protocol improves the best localized solutions, $R N G$ and $L M S T$ based broadcast oriented protocol (RBOP and $L B O P$ ) [1], [2] and is competitive with BIP.

This paper is organized as follows. In the next section, we present network and energy models. In Section III, we give a literature review of minimum energy broadcast protocols. In section IV, we present our computation of the theoretical optimal radius and the description of our broadcast algorithm. Section $\mathrm{V}$ presents the results of our simulations where we demonstrate the efficiency of our localized algorithm. The last section presents conclusion and future directions.

\section{PRELIMINARIES}

\section{A. Communication Model}

A wireless ad hoc network can be represented by a graph $G=(V, E)$ where $V$ is the set of nodes (hosts) and $E \subseteq V^{2}$ the edge set which gives the available communications: $(u, v)$ belongs to $E$ means that $u$ can directly send messages to $v$. In fact, elements of $E$ depend on the positions and the communication ranges of the nodes. Let us assume that the maximum range of communication, denoted by $R$, is the same for all vertices and that $d(u, v)$ is the Euclidean distance between nodes $u$ and $v$. The set $E$ is then defined as follows:

$$
E=\left\{(u, v) \in V^{2} \mid u \neq v \wedge d(u, v) \leq R\right\}
$$

So defined graph is known as the unit graph, with $R$ as its transmission radius. Every node $u \in V$ must be assigned an unique identifier $(i d)$. We also define the neighbor set $N(u)$ of the vertex $u$ as

$$
N(u)=\{v \mid(u, v) \in E\} .
$$

The neighborhood function is naturally extended to set of nodes: for a given subset $A$ of $V$, we have $N(A)=$ $\cup_{u \in A} N(u)$. The degree of a given node $u$ is the number of nodes in $N(u)$. The density of the graph is the average degree of each node.

We consider networks composed of nodes that can change the radius of their transmissions. In this case, the range of a node $u \in V$ represents the maximal distance between $u$ and a node which can receive its transmission. The range of a node $u \in V$ is denoted by $r(u)$ (with $0 \leq r(u) \leq R$ ). The graph induced by the range assignment function $r$ is denoted by $G_{r}=\left(V, E_{r}\right)$ where the edge set $E_{r}$ is defined by:

$$
E_{r}=\left\{(u, v) \in V^{2} \mid u \neq v \wedge d(u, v) \leq r(u)\right\} .
$$

\section{B. Energy Model}

In the most commonly used energy model, the measurement of the energy consumption of network interfaces when transmitting a unit message depends on the range of the emitter $u$ :

$$
E(u)=r(u)^{\alpha},
$$

where $\alpha$ is a real constant greater or equal than 2 and $r(u)$ is the range of the transmitting node. This model is used in [6].

In reality, however, it has a constant to be added in order to take into account the overhead due to signal processing, minimum energy needed for successful reception, MAC control messages and also possible overhead due to retransmission probability [7]. The general energy consumption formula is then:

$$
E(u)= \begin{cases}r(u)^{\alpha}+c & \text { if } r(u) \neq 0 \\ 0 & \text { otherwise }\end{cases}
$$

For instance, Rodoplu and Meng [8] consider the model with $E(u)=r(u)^{4}+10^{8}$. This last model, also used in [1], [9], is more realistic.

The measurement of the total power consumption is given by the following formula:

$$
E=\sum_{u \in V} E(u) .
$$

\section{RELATED WORK}

Topology control protocols aim to minimize the needed radius for a transmission, while preserving the connectivity of the network. Clementi, Ferreira, Penna, Perennes and Silvestri showed in [10] that this minimum energy range assignment problem is a NP-hard one. Wieselthier, Nguyen and Ephremides defined in [6] a topology control algorithm based on the MST subgraph, which is used to determine the transmission range of nodes: a node selects the transmission power that permits to cover all its neighbors in this subgraph. As the MST is always connected, the new graph derived from the new range assignment is also connected. In [1], the authors propose to use the $R N G$ as a connected subgraph instead of the $M S T$, the obvious advantage being that the $R N G$ can be computed in a totally local fashion. Li, Hou and Sha in [5] propose a new algorithm to compute a Local Minimum Spanning Tree (LMST) that keeps connectivity. Fig. 1 gives an example of an unit graph and its associated $M S T, R N G$ and LMST subgraphs.

Wieselthier, Nguyen and Ephremides proposed in [6] two other globalized greedy heuristics for the minimum-energy broadcast problem. They are called $B L U$ and BIP, and belong to the family of broadcast oriented protocols. The $B L U$ heuristic (Broadcast Least-Unicast-Cost) applies the Dijkstra's algorithm, while BIP (Broadcast Incremental Power) is a variant of the Prim's algorithm that uses the broadcast nature of wireless transmissions. Although the authors used an energy model with constant $c=0, B I P$ fits well with the general 


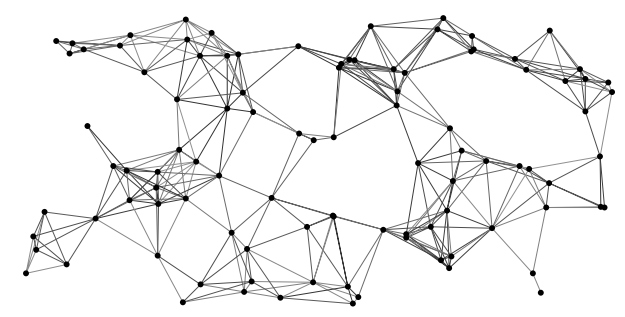

(a) Unit Graph

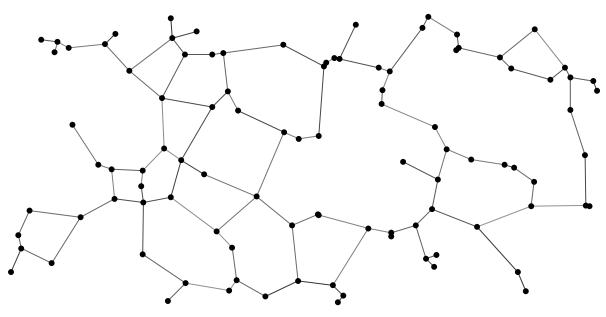

(c) Relative Neighbourhood Graph

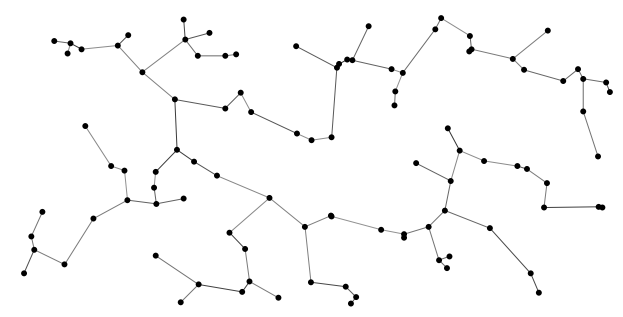

(b) Minimum Spanning Tree

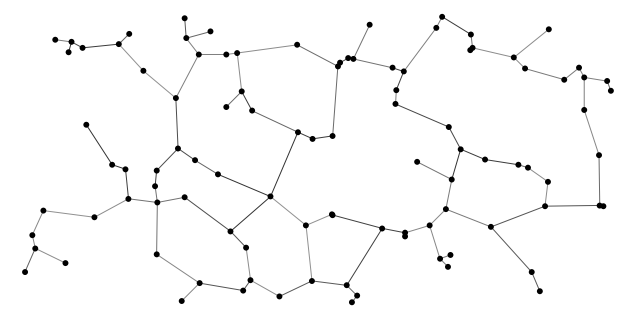

(d) Local Minimum Spanning Tree

Fig. 1. Example of an unit graph with its subgraphs (100 nodes with an average degree of 14).

model with an arbitrary constant. Some improvements of $B I P$ have since been proposed but always in a globalized manner and with an energy model using constant $c=0$ ( [11]-[13]).

In [1], we gave a localized broadcasting algorithm based on the RNG called RBOP for RNG Broadcast Oriented Protocol. The main idea of this protocol is based on the fact that covering only $R N G$-neighbors is enough to guarantee a full coverage of the network, while minimizing the energy consumption. The principle is quite similar to a Neighbor Elimination Scheme (NES) [14], [15] limited to $R N G$-neighbors with a radius adjustment to uncovered nodes. In the NES protocol, each node removes from an internal list the neighboring nodes that are supposed to have already received the message. The retransmission is canceled if the list becomes empty. In the original version of $R B O P, N E S$ is triggered only for message received by a non- $R N G$-neighbor while the message is immediately forwarded when it is received from a $R N G$-neighbor. It is shown in [2] that the protocol gives better performance (around $10 \%$ less energy consumption) when NES is performed in both cases. Moreover, the use of LMST instead of $R N G$ leads to a more efficient localized protocol. The variant based on LMST is called $L B O P$ for LMST Broadcast Oriented Protocol and is also described in [2]. This last version of the algorithm improves performances of $R B O P$ by approximately $20 \%$.

In [3], $\mathrm{Li}$ and $\mathrm{Hou}$ independently also proposed to apply LMST structure for broadcasting, and analytically concluded that multihop broadcast is more power efficient than single message with full transmission power when $\alpha \geq 2.2$. This article challenges the generality of such conclusion independently on constant $c$, which tends to suggest that it is always best to minimize the transmission radius to the minimal one, decided by LMST structure.

To the best of our knowledge, the idea about existence of an optimal 'target' radius for broadcasting is new, and our solution is both very elegant and experimentally confirmed.

\section{TARGET RADIUS BROADCAST ALGORITHM}

\section{A. Optimal Radius}

To introduce the idea of optimal radius, we will consider a geometrical area $S$ on which some nodes are to be placed. They are able to emit messages, with an arbitrary range that we have to determine, the goal being here to broadcast a message to the entire area, while minimizing the needed energy to achieve this.

We first have to place the relaying nodes. To do this, we choose an hexagonal tiling, that is the area is divided into several hexagons of side $r$, and nodes are placed on vertices. The quantity of vertices (i.e. nodes) depends obviously of $r$, which is also the range of the nodes. Fig. 2 shows the tiling for two different values of $r$.

We then have to choose the radius of the nodes, i.e. the value of $r$. We could choose a big radius, which would cover a large part of the area, or a smaller one, to cover just a small part of it. In the first case, we would need only a few emitting nodes, while in the second one, we would need a lot more of them to relay the message. This is like studying a mesh network where base stations are placed in an hexagonal way. To compute the optimal distance of emission, we are going to search the optimal distance between two emitting nodes on our hexagonal grid, that is the value of $r$ that minimizes the energy consumption when a broadcast occurs.

Knowing $r$ is the exact distance between two emitting nodes, we can easily compute the necessary quantity of them to cover the entire area. To do this, we just have to find how many hexagons, denoted by $h$, fit on our area of surface $S$ :

$$
\mathrm{h} \simeq \frac{\text { Surface of the area }}{\text { Surface of an hexagon }}=\frac{2 S}{3 r^{2} \sqrt{3}} .
$$

Since we place two nodes per each hexagon, the number of emitting nodes $n=2 h$ is then: 

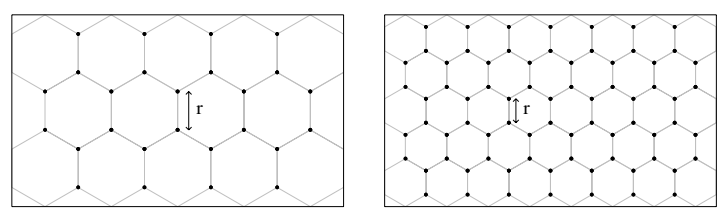

Fig. 2. Hexagonal tiling of the surface $S$ for different ranges $r$.

$$
n=\frac{k}{r^{2}}, \quad k=\frac{4 S}{3 \sqrt{3}} .
$$

The power consumption of a broadcast over this area is then:

$$
P C(r)=\left(r^{\alpha}+c\right) \frac{k}{r^{2}} .
$$

To find the optimal radius $r$, we just have to compute minimas of this function. Given that $\alpha \geq 2, c \geq 0$ and $r>0$, there are only a few cases we can enumerate:

- $\alpha=2, c=0$ : we have $P C(r)=k$ and this value does not depend on $r$;

- $\alpha=2, c \neq 0$ : we have $P C(r)=k\left(1+c . r^{-2}\right)$. There is no minima, but the greater $r$ is, the smaller the consumption is;

- $\alpha>2, c=0$ : we have $P C(r)=k r^{\alpha-2}$ which does not have a minima when $r>0$, but the smaller $r$ is, the smaller the consumption is;

- $\alpha>2, c \neq 0$ : we have $P C(r)=k\left(r^{\alpha-2}+c r^{-2}\right)$ which has a minima when its derivative reaches zero. We have:

$$
P C^{\prime}(r)=k\left((\alpha-2) r^{\alpha-3}-2 c r^{-3}\right),
$$

which reaches zero when :

$$
r=\sqrt[\alpha]{\frac{2 c}{\alpha-2}} .
$$

We can easily prove that it is a minima. Fig. 3 clearly shows that when $\alpha=4$ and $c=10^{8}$, the optimal radius is 100 . Below this value, there are too many emitting nodes, making the constant $c$ a problem while a radius greater than 100 makes the constant $\alpha$ a problem.

We can note that when $\alpha=2$, we have special cases. In the first case $(c \neq 0)$, it is better to emit at the maximal range,

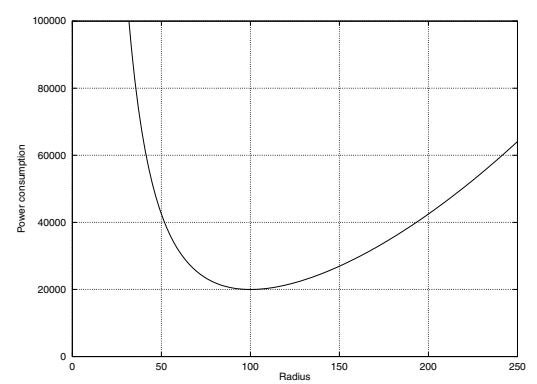

Fig. 3. Power consumption vs. chosen radius with $\alpha=4$ and $c=10^{8}$
TABLE I

TheORETICAL OPTIMAL EMITTING RADIUS $R_{o p t}$ FOR EACH $\alpha$ AND $c$.

\begin{tabular}{c||c|c}
$R_{\text {opt }}$ & $c=0$ & $c \neq 0$ \\
\hline \hline$\alpha=2$ & $-^{\dagger}$ & $R$ \\
\hline$\alpha>2$ & 0 & $\sqrt[\alpha]{\frac{2 c}{\alpha-2}}$.
\end{tabular}

$\dagger$ when $\alpha=2$ and $c=0$, the radius has no influence, we use 0 as an arbitrary value.

while in the second one $(c=0)$ the chosen radius does not influence the power consumption.

Given all these results, we can compute theoretical optimal values of the transmission radius, in an ad hoc network with a maximum range $R$. They are presented in table I for each possible value of $\alpha$ and $c$. With these theoretical values, we can place the optimal number of nodes on our area $S$ with an optimal distance between them, in order to have a minimal power consumption when a broadcast occurs.

\section{B. Target Radius LMST Broadcast Oriented Protocol}

While in a real ad hoc network we cannot decide the position of the nodes, it is possible to control the topology by changing their radii. Roughly, our goal here is to choose for each node a radius as close as possible to the optimal one.

To do this, we use $\angle B O P$ as a base broadcast algorithm for different reasons: it is localized, only nearest neighbors are monitored for the NES and it performs well when compared to the best known centralized protocol BIP. Its principle is as follows. Each node that receives the broadcast message for the first time generates a list of its $L M S T$-neighbors that have not received this message and starts monitoring the communications that occur in its neighborhood. Each time one of its $L M S T$-neighbors receives the message, this node is removed from the list. After a given timeout, two cases can happen: if the list is empty, the retransmission is canceled, otherwise the message is relayed with the radius needed to cover the furthest neighbor in the list.

As explained previously, it is not always optimal to rebroadcast with a minimal radius, because a too short radius will require more nodes to act as relays. To include the concept of optimal radius in $\angle B O P$, we modify some parts of this protocol, so that each node increases its radius up to the optimal one when a retransmission is needed. This variant of LBOP is called TR-LBOP for Target Radius LMST Broadcast Oriented Protocol.

Each node has to manage two lists $L$ and $L^{\prime}$ during the $N E S$. The first one, $L$, stores the neighbors needed to keep the connectivity of the network. As with $L B O P$ we use the $L M S T$-subgraph, so $L$ is defined by:

$$
\forall u \in V \quad L(u)=N_{L M S T}(u) .
$$

The list $L^{\prime}$ stores every other neighbors of the node, and is defined by:

$$
\forall u \in V \quad L^{\prime}(u)=N(u) \backslash L(u)
$$


During the NES, each neighbor that receives the broadcast message is removed from the corresponding list ( $L$ if it is a $L M S T$-neighbor, $L^{\prime}$ otherwise). Of course, the node $u$ can immediately remove the neighbor from which it received the message and their common neighbors which also received the same message, based on transmission radius used.

When the timeout is up, two cases can happen:

- The list $L$ is empty, in which case the retransmission is not needed to keep the connectivity. The reemission is canceled, as with $\angle B O P$.

- There is at least one node in $L$. In this case, the node $u$ has to rebroadcast the message to reach the nodes left in $L$.

In the second case, when the retransmission is needed, we know that the node will have to support the cost of the constant $c$ in the energy model. So, as explained previously, it can be more clever to increase the needed radius up to the optimal one, when it is possible. We define two values, $D_{L}$ and $D_{L^{\prime}}$. The first one, $D_{L}$ is defined by:

$$
D_{L}=\max \{d(u, v) \mid v \in L(u)\},
$$

$d(u, v)$ being the Euclidean distance between $u$ and $v$. The second one, $D_{L^{\prime}}$ is defined by:

$$
\begin{aligned}
D_{L^{\prime}} & =\left\{d(u, v) \mid v \in L(u) \cup L^{\prime}(u) \wedge\right. \\
\delta_{u v} & \left.=\min \left\{\delta_{u w} \mid w \in L(u) \cup L^{\prime}(u)\right\}\right\}
\end{aligned}
$$

where $\delta_{u v}=\left|d(u, v)-R_{o p t}\right|$.

In other words, the chosen distance is the length of the edge between the node $u$ and its non-reached neighbor which is the nearest one from $R_{\text {opt }}$. The final radius chosen is simply:

$$
r(u)=\max \left\{D_{L}, D_{L^{\prime}}\right\} .
$$

This modification leads to a situation where nodes mostly emit with a radius as close as possible to $R_{o p t}$. The increased number of reached neighbors is balanced with the full neighbor elimination scheme of $L B O P$, so the number of relays does not increase dramatically.

Performances evaluation is given in the next section.

\section{Performances}

In the simulations, we compare our protocol with $\angle B O P$ and BIP. $\angle B O P$ is an obvious choice, as ours is based on it, while $B I P$ has been chosen because it is the best known centralized protocol. As our protocol is localized, we can treat the performances of $B I P$ as the limit one can reach. We use the energy model proposed by Rodoplu and Meng [8] ( $\alpha=4$, $c=10^{8}$ ), that is the power consumption of an emission with a radius $r$ is given by:

$$
P C(r)=r^{4}+10^{8} .
$$

The parameters of our simulations are the following. The network is static and is always composed of 200 nodes

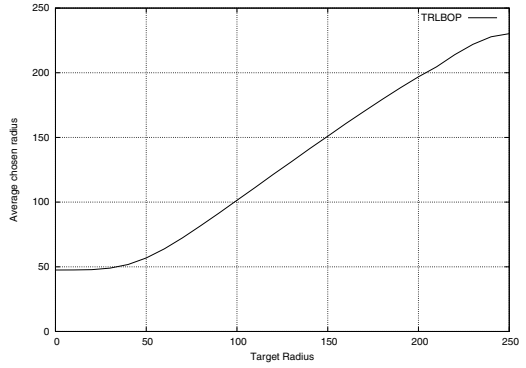

Fig. 4. Average chosen radius vs. target radius (density 50).

randomly placed in a square area whose size is computed to obtain a given density. The maximum communication radius $R$ is fixed to 250 meters. The MAC layer is assumed to be ideal, that is no collisions occur when two neighbors emit simultaneously. The timeout used in the neighbor elimination scheme is randomly generated. For each measure, 500 broadcasts are launched and for each broadcast, a new network is generated. Only connected networks are kept.

To compare the different protocols, we observe the total power consumption over the network when a broadcast has occurred. For each broadcast, we compute the total energy consumption:

$$
E_{\text {total }}=\sum_{u \in V} E(u)
$$

where $E(u)$ is the power consumption of $u$ that depends of the transmission radius as explained above. This total energy consumption $E$ is compared with the total energy consumption needed for the blind flooding protocol with maximal range:

$$
E_{\text {flooding }}=n \times\left(R^{\alpha}+c\right) .
$$

Finally we can compute the average Expanded Energy Ratio $(E E R)$ which is defined by:

$$
E E R=\frac{E_{\text {total }}}{E_{\text {flooding }}} \times 100 .
$$

In the simulations, we use as a parameter a value named Target Radius, which is the value used for $R_{o p t}$ in the computations. As shown on Fig. 4, TR-LBOP offers a way to control the radius of transmission by modifying this parameter. We can note that when a too short target radius is given to the nodes, they choose a bigger one that preserves the connectivity, which is insured by the use of $L M S T$. When a target radius of 0 is used, the nodes choose an average radius near to 50, which seems to be the minimal range needed for connectivity with the LMST in a network with density of 50 .

Fig. 5 shows the average EER obtained with a density of 50 for different values of $R_{\text {opt }}$. The most important observation is the existence of a minima, which proves the validity of our process. There really exists a radius for which the power consumption is minimal. Our protocol improves $L B O P$ by 


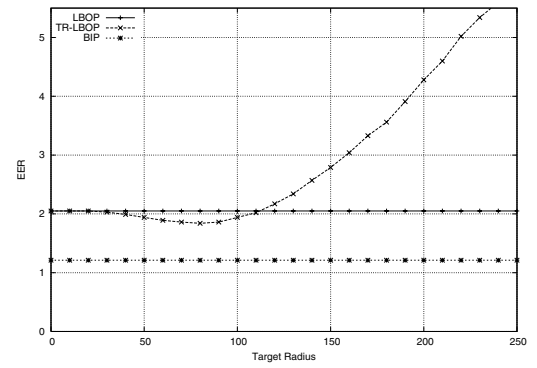

Fig. 5. EER vs. target radius (density 50).

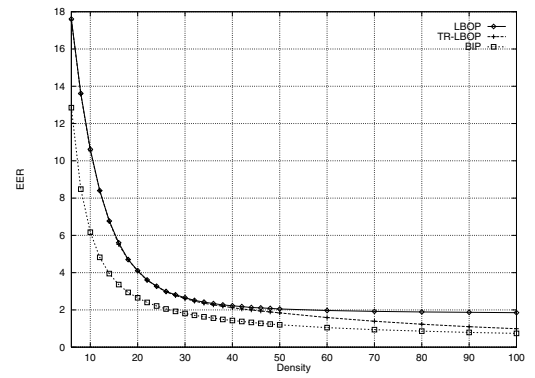

Fig. 6. EER vs. density.

about $10 \%$ with the use of this optimal radius, for the considered density. When compared with BIP, this improvement leads to a decrease of the overhead due to the decentralized computations. The experimental optimal radius observed here seems to be around 80 meters, which is near the theoretical value of 100 meters (see Fig. 3). The small difference can be attributed to various border effects.

Based on the computations for many densities, we give in Fig. 6 the best EER obtained for each of these densities. We can notice that $T R-L B O P$ becomes much more energy-efficient than $\angle B O P$ with higher densities, where the performances of $T R$ - LBOP can be compared to BIP. We give also in Fig. 7 the overhead in the energy consumption compared to BIP. A value of 150 for $\angle B O P$ and for a density of 100 means that the energy consumption of $\angle B O P$ is $150 \%$ higher than the consumption of $B I P$ for this density. We can notice that while the overhead is constantly increasing for $\angle B O P$ past a given density (around 30), the overhead of TR-LBOP is strongly decreasing past the density of 50 .

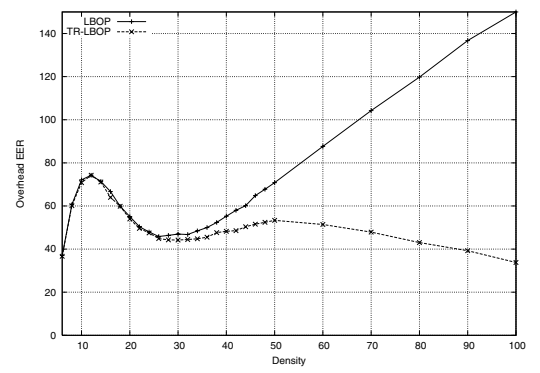

Fig. 7. Overhead EER vs. density.

\section{CONCLUSION}

In this paper, we introduced the concept of optimal radius, computed with an hexagonal tiling of the network area. We also proposed an energy efficient broadcast protocol named $T R-L B O P$ which is based on $L B O P$ and makes use of this optimal radius. Experimental results proved the existence of this optimal range when using $T R-L B O P$. The results show that this new protocol is energy-efficient and can be compared to the centralized protocol $B I P$, although all the computations are made locally by each node.

As future works, we want to consider different computation of the timeout used by the neighbor elimination scheme. We used here a random timeout while it could be more clever to favor certain nodes which could be more interesting than others. A more complex computation of the timeout could lead to further improvements in the energy consumption.

\section{REFERENCES}

[1] J. Cartigny, D. Simplot, and I. Stojmenović, "Localized minimum-energy broadcasting in ad hoc networks," in Proceedings of the IEEE Infocom 2003, San Francisco, USA, April 2003.

[2] J. Cartigny, F. Ingelrest, D. Simplot-Ryl, and I. Stojmenović, "Localized LMST and RNG based minimum-energy broadcast protocols in ad hoc networks," Ad hoc Networks, 2004, to appear.

[3] N. Li and J. Hou, "BLMST: A scalable, power-efficient broadcast algorithm for wireless sensor networks," 2003, submitted.

[4] G. Toussaint, "The relative neighborhood graph of finite planar set," Pattern Recognition, vol. 12, no. 4, pp. 261-268, 1980.

[5] N. Li, J. C. Hou, and L. Sha, "Design and analysis of an MST-based topology control algorithm," in Proceedings of the IEEE Infocom 2003, San Francisco, USA, April 2003.

[6] J. Wieselthier, G. Nguyen, and A. Ephremides, "On the construction of energy-efficient broadcast and multicast trees in wireless networks," in Proceedings of the IEEE Infocom 2000, Tel Aviv, Israel, 2000, pp. 585 $-594$.

[7] L. Feeney, "An energy-consumption model for performance analysis of routing protocols for mobile ad hoc networks," ACM Journal of Mobile Netwoks and Applications, vol. 3, no. 6, pp. 239-249, 2001.

[8] V. Rodoplu and T. H. Meng, "Minimum energy mobile wireless networks," in Proceedings of the IEEE International Conference on Communications (ICC'98), vol. 3, Atlanta, USA, June 1998, pp. 1633 $-1639$.

[9] X.-Y. Li and P.-J. Wan, "Constructing minimum energy mobile wireless networks," ACM Mobile Computing and Communication Reviews, vol. 5, no. 4, pp. 55-67, 2001.

[10] A. Clementi, A. Ferreira, P. Penna, S. Perennes, and R. Silvestri, "The minimum range assignment problem on linear radio networks," in European Symposium on Algorithms (ESA 2000), Sarbrüken, Germany, September 2000, pp. 143 - 154.

[11] T.Chu and I.Nikolaidis, "Energy efficient broadcast in mobile ad hoc networks," in Proceedings of the International Conference on Ad-Hoc, Mobile, and Wireless Networks (ADHOC-NOW'O2), Toronto, Canada, 2002, pp. 177-190.

[12] R. M. II, A. Das, M. El-Sharkawi, P. Arabshahi, and A. Gray, "Minimum power broadcast trees for wireless networks: Optimizing using the viability lemma," in Proceedings of the International Symposium on Circuits and Systems (ISCAS 2002), Scottsdale, USA, 2002, pp. 245248.

[13] P.-J. Wan, G. Calinescu, X.-Y. Li, and O. Frieder, "Minimum energy broadcast routing in static ad hoc wireless networks," ACM/Kluwer Wireless Networks, 2002.

[14] W. Peng and X. Lu, "On the reduction of broadcast redundancy in mobile ad hoc networks," in ACM MobiHoc 2000, Boston, Massachusetts, USA, August 2000, pp. $129-130$.

[15] I. Stojmenović, M. Seddigh, and J. Zunic, "Dominating sets and neighbor elimination-based broadcasting algorithms in wireless networks," IEEE Transactions on Parallel and Distributed Systems, vol. 12, no. 12, December 2001. 